\title{
Pharmacokinetics under the COVID-19 storm!
}

\author{
Venkatesh Pilla Reddy ${ }^{1}$, Eman Elkhateeb ${ }^{2}$, Heeseung $\mathrm{Jo}^{1}$, Natalie Natalie ${ }^{3}$, Emily \\ Lythgoe $^{1}$, Weifeng Tang ${ }^{4}$, Masoud Jamei ${ }^{5}$, Shringi Sharma ${ }^{6}$, and Amin Rostami-Hodjegan ${ }^{7}$ \\ ${ }^{1}$ AstraZeneca PLC \\ ${ }^{2}$ The University of Manchester Faculty of Medical and Human Sciences \\ ${ }^{3}$ AstraZeneca Pharmaceuticals LP \\ ${ }^{4}$ Affiliation not available \\ ${ }^{5}$ Certara UK \\ ${ }^{6}$ Acerta Pharma LLC \\ ${ }^{7}$ University of Manchester
}

June 28, 2020

\begin{abstract}
Aims: The storm-like nature of the health crises caused by COVID-19 has led to unconventional clinical trial practices such as the relaxation of exclusion criteria. The question remains: how can we conduct diverse trials without exposing sub-groups of populations to potentially higher drug exposure levels? The aim of this study was to build an extensive knowledge-base of the effect of intrinsic and extrinsic factors on the disposition of several repurposed COVID-19 drugs. Methods: Verified physiologically-based pharmacokinetic (PBPK) models were used study the effect of COVID-19 drugs PK in geriatric patients, race, organ impairment, DDI risks, disease-drug interaction for repurposed COVID-19 drugs. Furthermore, these models were used to predict epithelial lining fluid (ELF) exposure which is relevant for COVID-19 patients by accounting for the interplay between cytokines and metabolic disposition. Results: The simulated PK profiles suggest no dose adjustments are required based on age and race for COVID-19 drugs; however, sometimes dose adjustments are warranted for patients exhibiting hepatic/renal impairment in addition to COVID-19 co-morbidity. PBPK model simulations suggest ELF exposure to attain a target concentration was adequate for most drugs except azithromycin, atazanavir and lopinavir/ritonavir. Conclusion: We demonstrate that systematically collated data on the ADME, human PK parameters, DDIs, and organ impairment has enabled verification of simulated plasma and lung tissue exposure of many repurposed COVID-19 drugs to justify broader recruitment criteria for patients. In addition, developed PBPK model helped to assess the correlation between target site exposure to relevant potency values from in vitro studies for SARS-CoV-2.
\end{abstract}

\section{Title:Pharmacokinetics under the COVID-19 Storm}

Authors: Venkatesh Pilla Reddy ${ }^{1}$, Eman El-Khateeb ${ }^{2}$, Heeseung $\mathrm{Jo}^{1}$, Natalie Giovino ${ }^{3}$, Emily Lythgoe ${ }^{4}$, Shringi Sharma ${ }^{5}$, Weifeng Tang ${ }^{5}$, Masoud Jamei ${ }^{6}$, Amin Rastomi-Hodjegan ${ }^{2,6}$

${ }^{1}$ Modelling \& Simulation, Early Oncology, R\&D Oncology, AstraZeneca, Cambridge, United Kingdom;

${ }^{2}$ Centre for Applied Pharmacokinetic Research, University of Manchester, United Kingdom;

${ }^{3}$ Early Oncology Clinical, R\&D Oncology, AstraZeneca, United States;

${ }^{4}$ RED Biopharmaceuticals, AstraZeneca, United Kingdom;

${ }^{5}$ Clinical Pharmacology and Quantitative Pharmacology, RED, AstraZeneca, USA;

${ }^{6}$ Certara UK Limited, Simcyp Division, Sheffeld, United Kingdom 


\section{*Corresponding author:}

Dr. Venkatesh Pilla Reddy

Modelling and Simulation, Early Oncology, Oncology R\&D, AstraZeneca, Hodgkin Building, Chesterford Science Park, Little Chesterford, Cambridge, CB10 1XL, UK

Email: Venkatesh.Reddy@astrazeneca.com; Phone: +44 (0) 7742645481

Key words: COVID-19, Cytokine, ADME, M\&S, PBPK, DDI, Race, PKPD

Running title: Pharmacokinetics under the COVID-19 storm!

Word count: 4770 words

Abstract word count: 331

Figures/tables: 5 Figures, 3 tables ( $\max 8)$

Supplementary information: Supplementary Tables (Table S1, S2, S3, S4, S5), Supplementary figures (Figures S1, S2)

What is already known about this subject?

- Disease-drug interactions can result in exposure changes, which are well documented in cancer, cirrhosis, and rheumatoid arthritis. Cytokine storms are known to perturb the expression of CYP enzymes, conjugative enzymes, and transporters.

- Clinically observed pharmacokinetic data and the effect of race on repurposed COVID-19 drugs in geriatric COVID-19 patients are limited.

- There is some knowledge of the effect of intrinsic and extrinsic factors on the disposition of repurposed COVD-19 drugs, but dosing recommendations during cytokine storm are lacking

\section{What this study adds:}

- A comprehensive summary of ADME, DDI, Adverse Events (AEs) and simulated lung exposure for COVID-19 repurposed drugs

- Verified PBPK models that predict changes in drug exposure in various clinical scenarios to guide dosing information where clinical data are not possible, especially during this COVID-19 pandemic

- Simulated plasma and lung tissue exposure of drugs to justify broader recruitment criteria for patients and assess the relevant potency values from in vitro studies for SARS-CoV-2

\section{ABSTRACT}

Aims:

The storm-like nature of the health crises caused by COVID-19 has led to unconventional clinical trial practices such as the relaxation of exclusion criteria. The question remains: how can we conduct diverse trials without exposing sub-groups of populations to potentially higher drug exposure levels? The aim of this study was to build an extensive knowledge-base of the effect of intrinsic and extrinsic factors on the disposition of several repurposed COVID-19 drugs.

\section{Methods:}

Verified physiologically-based pharmacokinetic (PBPK) models were used study the effect of COVID-19 drugs PK in geriatric patients, race, organ impairment, DDI risks, disease-drug interaction for repurposed COVID-19 drugs. Furthermore, these models were used to predict epithelial lining fluid (ELF) exposure which is relevant for COVID-19 patients by accounting for the interplay between cytokines and metabolic disposition.

\section{Results:}


The simulated PK profiles suggest no dose adjustments are required based on age and race for COVID-19 drugs; however, sometimes dose adjustments are warranted for patients exhibiting hepatic/renal impairment in addition to COVID-19 co-morbidity. PBPK model simulations suggest ELF exposure to attain a target concentration was adequate for most drugs except azithromycin, atazanavir and lopinavir/ritonavir.

\section{Conclusion:}

We demonstrate that systematically collated data on the ADME, human PK parameters, DDIs, and organ impairment has enabled verification of simulated plasma and lung tissue exposure of many repurposed COVID-19 drugs to justify broader recruitment criteria for patients. In addition, developed PBPK model helped to assess the correlation between target site exposure to relevant potency values from in vitro studies for SARS-CoV-2.

\section{INTRODUCTION}

"Necessity is the mother of invention": Desperate times have often led to necessary efforts to satisfy an unmet need by unorthodox means. Clinical trials in drug development are no exception to this. In general, clinical trials are conducted in a systematic way and follow stringent recruitment criteria. Lack of diversity in patients recruited to drug trials was the subject of a recent draft Guidance for Industry by the US FDA, where they encouraged inclusion of the elderly, those at the extremes of the weight range, individuals with organ dysfunction, those with malignancies or certain infections such as HIV, and children [1]. This guidance emphasized the need to characterize drugs more comprehensively during early clinical development (e.g., with respect to drug metabolism and clearance pathways). In addition, the guidance indicated that, in many cases, dose adjustments can be made in specific populations to reduce significant differences in systemic exposure to the investigational drug. However, broadened recruitment of patients in COVID-19 drug trials has been an exception to the norm and is happening by default due to the speed of transmission of the virus. The pandemic has accelerated the adoption of the draft guidance in respect to broadening recruitment. However, the question of how this could be done without compromising sub-groups of populations with the burden of potentially higher exposure levels to drugs, especially for drugs that lack dedicated safety studies in these sub-population groups.

COVID-19 pandemic in perspective: Several therapeutic agents have been evaluated for the treatment of COVID-19, but remdesivir, acalabrutinib, ibrutinib, and dexamethasone have shown some positive results [2-4]. The FDA has issued guidance related to COVID-19 [5] which highlights the safety of trial participants as a key consideration for ongoing trials in addition to establishing the efficacy of drug against SARS-CoV-2. The estimated basic reproduction number for SARS-CoV-2 is 2.65 days (with a range from 1.85 to 3.41 days) [6]. High transmission rate of COVID-19 infection has led to changes in study design; trials with greater diversity in patient recruitment are becoming more common than those with strict protocol inclusion and exclusion criteria for patient characteristics. This rapid enrollment, however, may result in higher exposure levels of drugs in study subjects.

Drugs on Trial for COVID-19: As of May 21 ${ }^{\text {st }}, 2020$, there were 1,621 COVID-19 trials on clinicaltrials.gov, of which 912 are active and/or recruiting. To address the urgent need for therapeutic remedies for COVID19, efforts to repurpose existing drugs are increasing hence most trials are focused on existing drugs rather than Investigational New Drugs. These repurposed drugs consist of anti-virals (azithromycin, atazanavir, baloxavir, darunavir, lopinavir, remdesivir, ritonavir), anti-cancer drugs (acalabrutinib, ibrutinib, baricitinib, ruxolitinib), anti-inflammatory (dexamethasone), large molecules (siltuximab, emapalumab, tocilizumab), antimalarials (chloroquine, hydroxychloroquine), and one anti-diabetic and heart failure treatment drug (dapagliflozin). Azithromycin is dosed in combination with hydroxychloroquine.[7]

Typical COVID-19 Patient and Cytokine Storm: A typical hospitalized COVID-19 patient exhibits overproduction of early response pro-inflammatory cytokines such as tumor necrosis factor (TNF), interleukin-6 (IL-6), and interleukin-1 beta (IL-1 $\beta$ ). This is known as a 'cytokine storm,' and is thought to be one of the major causes of acute respiratory distress syndrome (ARDS) and multiple-organ failure [8] . Patients presenting with cytokine release syndrome typically show cytopenia, elevated creatinine, deranged coagulation 
parameters, and high C-reactive protein (CRP) [9]. The common symptoms and cascade of a COVID-19 induced cytokine storm [10] are depicted in Figure 1. Due to the relationship between cytokine release syndrome and poor outcomes among COVID-19 patients, many of the drugs (small molecules and biologicals) currently being tested aim to dampen this severe immune response, while others target the virus' ability to infect cells. Inflammation resulting from cytokine release starts locally, in areas such as pulmonary tissue, and spreads to other organs through systemic circulation. This triggers compensatory biological repair processes which restore tissue and organ function; however, these processes can lead to fibrosis and persistent organ dysfunction of the lungs, heart, liver and kidney [11]. Many small molecule drugs are metabolized by cytochrome P450 (CYP) enzymes typically expressed in the liver, gut, lung and kidney. Several proinflammatory cytokines (IL-1 $\beta$, IL-6, TNF- $\alpha$, IFN- $\gamma$ ) have been reported to downregulate the expression of these enzymes and transporters such as P-gp by inducing transcription factors (e.g. PXR, CAR, NF-kB, and $\mathrm{HNF}$ ), which in turn reduce the abundance of CYP450 and P-gp in tissues. This influences the absorption, distribution and metabolism of drugs, as represented in Figure 1.

Information on Study-sub populations: Remdesivir clinical trial [2] patient characteristics included chronic liver $(2 \%)$ and kidney disease $(6 \%)$, cancer $(8 \%)$ and obesity $(37 \%)$. $36 \%$ of patients were $>65$ years and patients of different race were included in the trial population. A large proportion of COVID-19 patients requiring hospitalization and treatment are geriatric and it is likely that this patient population may exhibit exposure differences. The possibility of testing the effect of intrinsic and extrinsic factors clinically is limited, for instance PK on the geriatric COVID-19 patients.

Physiologically-based pharmacokinetic (PBPK) models: The pharmacokinetic and ADME data for single or multiple doses is relatively abundant for COVID-19 repurposed drugs and can be used to build PBPK models. These models utilize three-components: a system component, a drug-dependent component, and a clinical trial-specific component. Details of human physiology are specified in system components, for instance drug metabolizing enzyme or transporter enzyme abundance, tissue blood flow to organs, and so on. Drug properties such as solubility, fraction metabolized by enzymes and renal clearance, are specified in the drug-dependent components, whilst the clinical trial-specific component defines the simulated scenario, including dosing regimen, population age, and gender proportion. PBPK models combine these components to predict drug ADME, and by extension pharmacokinetics (PK), in a simulated scenario where clinical study data are not available. These verified PBPK models can provide the ability to extrapolate beyond the available data and account for disease-drug interactions [12, 13].

Target site concentrations: As COVID-19 mainly targets lung tissue making it difficult to assess tissue concentration, understanding expected target concentration may be crucial, particularly when attempting to translate observed effect from the in vitro studies to projected clinical situation. PBPK models are useful to predict drug unbound concentrations in the epithelial lining fluid (ELF) [14] which is relevant for treatment of COVID-19 patients, as it can contain a persistent reservoir of virus.

The objectives of this study were to gather information on the ADME, PK/PD, DDI, AEs, and the effect of intrinsic and extrinsic factors on the disposition of all repurposed drugs currently under trial for COVID-19. This enabled us to explore the utility of PBPK models for possible clinical scenarios after verifying them based on the gathered pre-clinical and clinical data. Then we used the models to predict expected alterations in exposure to these drugs in sub-populations of COVID-19 patients which have not yet been studied for some of the drugs e.g., in patients with older age, different race, and hepatic/renal impairment. These variations were studied in relation to target site concentrations. All these models were developed by accounting for the interplay between cytokines and metabolic disposition.

\section{METHODS}

Clinical Pharmacokinetics and Pharmacodynamics data extraction of COVID-19 repurposed compounds and COVID-19 patient characteristics

Trials of drugs repurposed for COVID-19 were selected in this work from the American Society of Health- 
System Pharmacists (ASHP) list [15]. In addition, two drugs from AstraZeneca currently being studied in clinical trials are acalabrutinib, a Bruton tyrosine kinase (BTK) inhibitor [3], and dapagliflozin, a sodiumglucose transport protein 2 (SGLT2) inhibitor. Details of these and other repurposed drugs are summarized in detail in Table 1 and in Supplementary Text. These repurposed drugs were anti-virals (azithromycin, atazanavir, baloxavir, darunavir, lopinavir, remdesivir, ritonavir), anti-cancer drugs (acalabrutinib, baricitinib, ruxolitinib), anti-inflammatory and immunomodulators (dexamethasone), biologicals (siltuximab, emapalumab, tocilizumab), antimalarial (chloroquine, hydroxychloroquine), and anti-diabetic and heart failure treatment drug (dapagliflozin). Clinical studies for probe compounds were queried with appropriate literature searches and through the University of Washington Drug Interaction Database (UWDIDB). Dosing regimens, relevant PK parameters, DDI, adverse events, applicable demographic data, such as age, gender, race, PK in hepatic and renal impairment and genotype, were manually extracted from the selected publications. Cytokine information, such as IL-6 levels in a typical COVID-19 patient, was extracted from literature and compared with other cytokine storm disease conditions such as cancer, rheumatoid arthritis etc.

PBPK model development to study the effect of age, race and organ dysfunction

PBPK modeling and simulations were performed using Simcyp V19 simulator (Certara UK Ltd., Sheffield, UK) for clinical trial scenarios as shown in Supplementary Figure 1. Well established or fit-for-purpose PBPK models were verified against available clinical data before being utilized to simulate scenarios where no clinical data are available. PBPK models of acalabrutinib, baricitinib, ruxolitinib, ritonavir, darunavir and dapagliflozin were previously verified extensively and they have been submitted previously as part of new drug application submission to the FDA. The rest of the PBPK models (azithromycin, atazanavir, lopinavir, chloroquine, hydroxychloroquine) were reported in peer reviewed journals with reasonable level of verification. These verified PBPK compound files were obtained from either Simcyp Simulator compound library file or repository (https://members.simcyp.com/account/libraryFiles/) or were built using reported compound input parameters. The PBPK model input parameters are shown in Supplementary Table $\mathbf{1}$ and key drug parameters required for simulating lung concentration are shown inSupplementary Table 2 . Trial designs for all simulations were set to 10 trials of 10 patients with the relevant populations constrained by age range and the proportion of females which reflected the reported study design of clinical studies where available. Dosing amounts and schedules were chosen as per the respective compound dosing recommendations and administered as an oral single-dose or multiple-dose across all simulations. The predictive performance PBPK model of probe compounds was measured by the ratio of the mean predicted AUC to the mean observed AUC where possible (Supplementary Table 3 ). For organ dysfunction simulations, $\mathrm{AUC}_{\text {diseased }}$ to $\mathrm{AUC}_{\text {healthy }}$ ratios for both observed and predicted scenarios were calculated as predictive performance measures. The predictability was considered acceptable if this metric fell between the upper and lower limits (0.5 to 2fold of observed data). Differences in exposure from race in Caucasian, Japanese and Chinese populations were predicted using Simcyp's population library repository using the "Healthy-Volunteer," "Japanese," and "Chinese Healthy Volunteer" population models, respectively. Simulations for hepatic impairment were performed using Simulator's "Cirrhosis CP-A", "Cirrhosis CP-B", and "Cirrhosis CP-C" populations, while for renal impairment, populations "RenalGFR_30-60", and "RenalGFR_less_30" were used. The effect of age, race and organ impairment simulations were not simulated for baloxavir, remdesivir and large molecules, as adequate PBPK modelling was not possible due to limited data availability in the literature. Based on recent findings from IQ consortium [16] the impact of renal impairment can be well predicted within 2-fold of observed PK (AUC) and for hepatic impairment $>70 \%$ of the cases were predicted within 2-fold, supporting the utility of PBPK modeling for the study of organ dysfunction.

Simulating lung concentration for COVID-19 drugs

The permeability-limited lung model is particularly suitable for modeling and simulating drug disposition within the lung mass compartment and epithelial lining fluid compartment, which are the target sites for antiviral effects. The methodology of simulating these concentrations has been applied previously to tuberculosis [14], and recently for three COVID-19 repurposed drugs [17]. 


\section{PBPK simulation and comparison with observed clinical data}

The predicted $\mathrm{C}_{\max }$ and AUC in healthy subjects were compared to the observed data to ensure models were suitable for their intended use. Subsequently, the predicted $\mathrm{C}_{\max }$ and AUC ratios of organ dysfunction (estimated as the ratio of predicted $\mathrm{C}_{\max }$ and AUC of organ dysfunction relative to healthy populations) were compared with the observed organ dysfunction $\mathrm{C}_{\max }$ and AUC ratios. Partition coefficients $\left(\mathrm{K}_{\mathrm{P}}\right)$ between lung tissue and plasma data were available in rats (as shown in Supplementary Table 2 ). Rat tissue $\mathrm{K}_{\mathrm{P}}$ values were compared to in silico predicted human $\mathrm{K}_{\mathrm{P}}$ values, assuming the unbound distribution is independent of species.

\section{Sensitivity analyses with varying degrees of CYP suppression by cytokine storm}

Sensitivity analysis, via Simcypß's sensitivity analysis tool, was performed to study the effect of AUC change with reduced CYP3A4 abundance in the liver and gut. Acalabrutinib and ibrutinib are sensitive CYP3A4 substrates, so these were chosen for this sensitivity analyses. CYP3A4 abundance was varied, with values 10 to $90 \%$ lower than those observed in healthy volunteers. Physiological differences between healthy volunteers and patients with hepatic organ impairment are shown in Supplementary Table 4.

\section{RESULTS}

Cytokine levels in COVID-19 patients

Elevated cytokine levels alter the PK, and thus exposure, of small molecules. A few clinical studies have compared drug exposure before and after treatment with cytokines. The use of Interferon (IFN)- $\alpha$ in chronic hepatitis B has been found to decrease theophylline (metabolized mainly by CYP1A2) clearance by $50 \%$ and prolong the half-life of the drug by $70 \%$ [18]. Alternatively, blocking IL-6 receptor activity with drugs such as sarilumab and tocilizumab reduces the AUC and $\mathrm{C}_{\max }$ of simvastatin (CYP3A4 substrate) by $40-57 \%$ $[19,20]$.

As clinical studies addressing the impact of different drug metabolizing enzymes and transporters are not always feasible, in vitro models of hepatic and intestinal cell lines can be used. Exposure of hepaRG hepatic cells to IL-6 (10 ng/mL) for $72 \mathrm{~h}$ has been found to decrease CYP3A4 mRNA expression and activity by more than $80 \%$, CYP1A2 activity by $60 \%$, and CYP2B 6 and 2C19 activity by $80 \%$ [21]. In EpiIntestinal cells the expression of Phase I enzymes CYP2C19, CYP2C9, and CYP3A4 was reduced by 40-50\% (MAO A, 35\%; MAO B, 42\%), whilst activities of CYP2C19, CYP2C9, and CYP3A4 were suppressed by $20-75 \%$ following exposure to IL-6. However, it is worth noting that extrapolation of in vitro results to in vivo models is challenging due to the complexity of biological systems, disease pathophysiology, changes in cytokines levels, and interactions among cytokines and DME targets. One difficulty, for example, is making sure that IL-6 is added to cells in concentrations that mimic physiological levels in healthy and diseased states. In healthy individuals, serum IL-6 concentrations range from 1.3 to $10.3 \mathrm{pg} / \mathrm{ml}$, rising to 2.6 to $123 \mathrm{pg} / \mathrm{ml}$ in some patient groups with inflammatory diseases including: obesity [22] rheumatoid arthritis [23], psoriasis [24], and cirrhosis [25]. A recent meta-analysis reported IL-6 levels in COVID-19 patients with complex clinical complications to be nearly three-fold higher than in patients with few complications [8]. The average serum level of IL-6 across the 6 studies reported in this review was $65.5 \mathrm{pg} / \mathrm{ml}$ in patients with SARDS; severe acute respiratory distress syndrome, which is significantly higher than in non-severe cases of disease $(21.4 \mathrm{pg} / \mathrm{ml})$. Mortality rates correlated with higher levels of IL-6, and the addition of IL-6 inhibitors such as tocilizumab improved clinical outcomes with no deaths in a total number of 21 patients receiving treatment [8]. Similar results have been observed for acalabrutinib in COVID-19 patients; elevated IL-6 levels [median (range) of $44(25-89.8) \mathrm{pg} / \mathrm{mL}]$ were noted in COVID-19 patients with a significant $\left(\mathrm{p}=6.5 \mathrm{E}^{-4}\right)$ decline observed during acalabrutinib treatment. [3]

PK/PD of repurposed Covid-19 drugs

Table 1 summarizes the observed data of pharmacokinetics and pharmacodynamics properties of repurposed COVID-19 drugs. The half-maximal inhibitory concentration $\left(\mathrm{IC}_{50}\right)$ parameters obtained were treated as 
unbound $\mathrm{IC}_{50}$, as the experimental conditions utilize serum free conditions for COVID-19 drugs and this hypothesis was recently supported by Fan et al [26]. However, the in vivo plasma and lung tissue concentrations were corrected using in silico predicted tissue protein binding and measured plasma protein binding. The preclinical rat partition co-efficient $(\mathrm{Kp})$ values for lung tissue are compared with human predicted $\mathrm{Kp}$ values as listed in Supplementary Table 2 .

ADME, DDI and adverse events of repurposed COVID-19 drugs

Table 2 summarizes the main metabolizing enzymes, DDI, and adverse events of repurposed COVID-19 drugs. COVID-drugs being a perpetrator: No clinically significant DDIs are anticipated except ritonavir and darunavir; COVID-drugs being a victim: Weak to moderate DDI risk is anticipated for COVID drugs that are mainly metabolized by CYP3A4 except ibrutinib.

Effect of age and race on pharmacokinetics of repurposed COVID-19 drugs

Figure 2 shows the PBPK model simulated PK profiles for different age brackets: 18 to 40 years, 40 to 65 years, and 65 to 98 years (geriatric population). This age brackets represented a typical demographics from a recent remdesivir clinical trial [2]. The PBPK model-based results are in line with observed data as shown inSupplementary Table 3 . Though geriatric population exhibited higher exposure for all drugs compared to the age bracket of 18 to 40 years, but fell within $95 \%$ PI of healthy volunteers except for dexamethasone, which is marginally outside the shaded area of 95\% PI (Figure 2A). PBPK analysis suggests race has no clinically meaningful effect on the clearance of all COVID-19 drugs, as the mean simulated Chinese and Japanese population profiles fall within 95\% PI of Caucasian population (Figure 2B) Therefore, no dose adjustment is required based on race.

Effect of organ dysfunction on PK of repurposed COVID-19 drugs

The role of cytokines on the disposition of anti-cancer and rheumatoid arthritis drugs is well understood; however, this this has not been evaluated in COVID-19 patients. Figure 3 and Table 3show how hepatic impairment resulting from cirrhosis and renal impairment, change the exposure levels of these repurposed drugs. The absolute expression of CYP3A levels in severe cirrhotic subjects is assumed to be the same as in COVID-19 patients (seeSupplementary Tables $\mathbf{4}$ and $\mathbf{5}$ for physiological differences between Healthy population and either liver cirrhosis or renal impairment populations within Simcyp PBPK Simulator V19). The exposure of azithromycin, atazanavir, acalabrutinib, ruxolitinib, and dexamethasone was clearly higher in severe hepatic impairment (Figure 3A ) relative to healthy volunteers, suggesting the need for dose adjustment. In case of renal impairment (Figure 3B), ruxolitinib, atazanavir and azithromycin plasma exposures were remarkably higher in severe conditions relative to healthy population. Comparing predicted AUCR ( $\mathrm{AUC}_{0-[?]}$ in hepatic impairment/ $\mathrm{AUC}_{0-[?]}$ in healthy volunteers) with their corresponding observed values (whenever available) shows consistency within 2-fold of observed data for all drugs.

Simulating target site concentration for COVID-19 drugs

Figure 4 shows the simulated relative exposure of lung to plasma at steady state, depicting unbound epithelial lining fluid concentrations along with $95 \%$ prediction intervals. The horizontal dashed lines show $\mathrm{IC}_{50}$ and $\mathrm{IC}_{90}\left(9 \times \mathrm{IC}_{50}\right)$ range of target inhibition. The input parameters used for the permeability-limited lung model [14] are shown inSupplementary Table 1 and Supplementary Table 2.Overall, all COVID19 drugs appear to reach adequate exposures at steady-state over the target $\mathrm{IC}_{50}$ values of respective drugs and stays above for minimum of $8 \mathrm{hr}$ for all drugs except for all anti-viral drugs where reported $\mathrm{IC}_{50}$ values against SARS-CoV-2 were reported [27]. Nevertheless, these anti-virals exposure were above $\mathrm{IC}_{50} / \mathrm{IC}_{90}$ if human immunodeficiency virus (HIV) target values were used. Achieving $90 \%$ of target inhibition with unbound ELF concentration in most of the patients is achievable for a period of time ( $>4$ hrs; Figure 4 ) except for all anti-viral drugs. Total (parent + metabolite) unbound plasma and ELF concentration were used to estimate an average BTK occupancy for acalabrutinib (a covalent BTK binder). Estimated BTK occupancy was $>95 \%$, is consistent with the observed effectiveness of acalabrutinib in COVID-19 patients (NCT04346199) [3]. 
It has been proposed that the virus is internalized by receptor mediated endocytosis and delivered to lysosomes where it replicates. Some of these drugs have shown efficacy in raising the lysosomal PH (lysosomotropic drugs), resulting in lysosomal trapping of the virus and preventing its spread within the cell. This in theory might help the drug to be concentrated at the target site and thus, lower doses of the drug could to be required to achieve therapeutic efficacy. The lysosomotropic potential for COVID-19 drugs were predicted based on Ufuk et al., [28] and shown in Supplementary Table 2 . This lysosomotropic potential of chloroquine, hydroxychloroquine, atazanavir and remdesivir were appeared to be beneficial for attaining a required target exposure and efficacy. However increased exposure might also have consequences towards side effects.

\section{Sensitivity analyses with varying degrees of CYP suppression by cytokine storm}

The relative change in exposure (AUC) for CYP3A substrates such as acalabrutinib and ibrutinib when CYP3A expression reduced to one tenth of its healthy level in both the gut and liver is shown inFigure 5 . As a worst-case scenario, reducing $90 \%$ of CYP3A4 abundance in liver and gut, resulted in $\sim 4$-fold increase in AUC for acalabrutinib, while it was 15-fold change for ibrutinib.

\section{Discussion}

Whilst efforts for developing vaccines against COVOD-19 is intensively continuing, there are no indications that any of the programs will provide a safe and effective vaccine at the scales needed to reverse the pandemic caused by the virus before the end of 2020. Public health measures have been successful to various degrees in different countries in reducing the rate of infection. However, as these measures ease off to balance the negative economic prospects of lockdowns and social distancing, many expect that spread of the virus will continue albeit at lower rate even if we avoid a second peak. Therefore, ability for therapeutic management of patients who are infected remains a viable and necessary element of fight against the pandemic in parallel to the development of vaccines and public health measures to control the spread.

Great advances have been made in the use of modeling tools to predict and optimize doses and dosing schedules for clinical trial optimization and to inform drug labels [13, 29-31]. The current situation surrounding COVID-19 requires fast decision in clinical trial design with limited information, consequently, resulting in a potentially higher risk or lower benefit. A few such examples include higher incidence of side effects for chloroquine and lack of benefit from lopinavir and ritonavir combination therapy [32, 33]. COVID patients who received chloroquine with azithromycin experienced QTc prolongation, which may be attributed to PK or PD changes in target tissues as a result of DDI. To help drug developers and regulators surrounding the development of candidate treatments and regimens for Covid-19, reliable and effective modelling tools for quantifying and comparing therapy options, particularly in cases where clinical data are scant, will be very useful.

For typical drug development, in theory, the effect of all extrinsic and intrinsic patient factors can be tested clinically. However, ethical and practical issues limit the numbers of feasible studies one can conduct. As shown in Supplementary Figure 1, there are more than 100 untested clinical scenarios for COVID-19 repurposed drugs, due to limited time and resources. In this current COVID-19 pandemic, PKPD and PBPK modeling tools are already helping to optimize and accelerate candidate therapeutics [26, 32].

PBPK modeling approaches integrate drug data and physiological or system data together with patient factors, to predict certain untested situations using current knowledge. In recent years there have been several examples where PBPK modelling has been used in lieu of many clinical studies [30]. Currently, there is a lack of knowledge regarding intrinsic and extrinsic factors on the disposition of repurposed drugs for COVID-19. Therefore, in this work, we a) present a summary of ADME, PK/PD, DDI; b) provide a repository of PBPK models, input parameters to simulate the unbound plasma and/or lung epithelial lining fluid concentrations; and c) utilize PBPK models to predict expected alterations in exposure to these drugs in sub-populations of COVID-19 patients which have not yet been studied for some of the drugs in patients with older age, different race, and hepatic/renal impairment. The drugs selected for this analysis relied on 
verified PBPK model availability, and robust in vitro and clinical evidence of their effects on drug clearance.

Creating innovative solutions for dosing optimization under time constraints comes with uncertainty and gaps in knowledge which can only be addressed as more data becomes available. We can, however, prioritize available resources to reduce the number of patients-at-risk due to sub-optimal dosing and dosing-schedules, by simulating the situations where no clinical data are available. In this way, we can establish a scientific factbase and triaging process, to accelerate our understanding of therapeutic interventions for COVID-19. To facilitate knowledge-sharing and advance the field, modelling workspaces can be uploaded to an open-access, modelling tool platform members' area or Github (http://github.com/). Supplementary Files (workspaces) andSupplementary Table 1 provide PBPK models that can be readily accessed by scientists for use in their own research.

Table 1, 2, and 3 provide PK, PD, DDI and clinical pharmacology information for COVID-19 drugs. No clinical DDI data are available in many instances for example, combination of ritonavir (a CYP3A reversible and Time dependent inhibitor) with BTK inhibitors such as ibrutinib and acalabrutinib. Based on PBPK modeling -the DDI risk appears to be higher with ibrutinib when combined with ritonavir (AUC ratio of 44-fold), while ritonavir combination increased acalabrutinib AUC by 5.7 -fold); however, no DDI risk is anticipated for dapagliflozin, as it is mainly eliminated by UGT1A9 substrate.

As many of the repurposed drugs studied in this paper are primarily metabolized in the liver, it was unsurprising to find that impaired liver function or liver disease impacts the PK of these drugs. Baricitinib is mainly excreted unchanged by the kidney and thus its PK can be altered in renal failure conditions. Figure $\mathbf{3}$ andTable $\mathbf{3}$ and $\mathbf{4}$ shows the simulations for COVID-19 drugs under organ impairment situations. PBPK models suggests dosing adjustment for CYP3A substrates like ibrutinib, dexamethasone, and acalabrutinib likely to be necessary.

Sensitivity analyses can be a useful tool, to study the uncertainty in model input parameter and assess its impact on simulated PK profiles. For instance, in rheumatoid arthritis and cancer, it was reported that CYP3A4 content in the liver and gut was reduced by $\sim 30 \%$ [12] but such meta-analyses and reports are not yet available for COVID-19. Automated sensitivity analysis using Ibrutinib and acalabrutinib as a tool compounds suggest around 15-fold and 4-fold increase in AUC, if the CYP3A levels are reduced by $90 \%$ from normal levels (Figure 5 ), respectively.

PBPK enables better handling of tissue site concentrations compared to population-based PK modelling, and we can account for disease-drug interaction i.e. cytokine storm. In this study we utilize in vitro $\mathrm{IC}_{50}$ rather than in vivo: $\mathrm{EC}_{50}$ due to scarcity of this data. Nevertheless, when unbound in vivo $\mathrm{EC}_{50}$ data becomes available, we can easily update the exposure profiles with relevant target inhibition values and explore IVIVE as shown in Pilla Reddy et al., [34]

In vitro $\mathrm{IC}_{50}$ were generated for anti-viral drugs using serum free media in most instances, and thus we can assume in vitro $\mathrm{IC}_{50}$ are basically unbound values; however, in-vivo lung tissue and plasma profiles should be corrected for lung tissue protein binding and plasma protein binding respectively to obtain $\mathrm{K}_{\mathrm{P} \text {,uu }}$, under the assumption that the unbound drug is pharmacologically active [26].

Simulating the plasma and/or lung exposure of drugs used in COVID-19 therapies is of importance, as the unbound tissue concentration drives the efficacy and safety of a drug. Non-invasive imaging methods (PET/MRI) can be used to determine tissue drug concentrations; however, it is logistically challenging to routinely employ these methods during drug development. We therefore need high-throughput and costefficient methods to predict the tissue concentration of drugs. In order to do so, we must accurately predict drug distribution and clearance into and out of tissue. We hypothesize that lung concentrations of drugs can be predicted through in vitro to in vivo extrapolation, by incorporating the relevant parameters such as permeability and transporter data. Preclinical, quantitative, whole-body autoradiography distribution data could be used to understand the partition between plasma and lung tissue or organ of interest to some extent, assuming the unbound partition coefficient is independent of species. Quantitative whole-body autoradiography data in rats was available for most of the drugs (Supplementary Table 2 ) allowing 
comparisons with predicted total $\mathrm{K}_{\mathrm{P}}$ values within the Simcyp Simulator.

The permeability-limited lung model within the Simcyp Simulator has been used and verified with known anti-tuberculosis drug concentrations in epithelial lining fluid (ELF). This prediction of ELF unbound concentration is particularly relevant for COVID-19 patients, as distribution of COVID-19 drugs should be targeted into immune privileged sites like ELF in the lung, which may represent a persistent reservoir for the virus [11]. Highly ionizable drugs might experience different lung uptake due to changes in lung $\mathrm{pH}$ due to COVID-19, which were accounted for in our PBPK model. As shown in Figure 4, simulated unbound ELF profiles were compared with in vitropotency values that are thought to inhibit SARS-CoV-2, and most drugs exhibited reasonable duration of target inhibition.

In summary, in this study, we provide a database for relevant PK, PD, DDI and AEs attributes for ongoing COVID-19 treatment, including both small molecules and large molecules. Furthermore, we have highlighted the application of quantitative modelling tools that could be helpful to understand the intrinsic and extrinsic factors that can affect the PK of these repurposed small molecule drugs, including the strategy of simulating the plasma and/or ELF concentrations under physiological changes of COVID-19 population. Prospective PBPK modelling and simulations can identify gaps in available datasets and play a role in answering critical clinical questions related to patient intrinsic and extrinsic factors that can't be studied in the time-constrained COVID-19 pandemic.

\section{Acknowledgments}

The authors would like to thank the patients, their families, and the clinical teams who worked on the studies.

\section{Conflicts of Interest}

Venkatesh Pilla Reddy, Heeseung Jo, Natalie Giovino, Emily Lythgoe, Shringi Sharma and Weifeng Tang, are all employed by for AstraZeneca. Eman Elkhateeb is a Ph.D. student at University of Manchester. Prof Amin Rastomi and Masoud Jamei holds shares in Certara, a company focusing on Model-Informed Drug Development.

\section{Funding}

The study was supported by AstraZeneca. Eman is supported by $\mathrm{PhD}$ funding from the Egyptian government and CAPKR

\section{Author contributions}

All authors were involved in designing the studies and performing the study analyses. All authors wrote the manuscript. VPR, EE, and HJ performed the modelling analyses. All authors have approved the manuscript for submission.

\section{Data accessibility statement}

The authors confirm that the clinical data supporting the PBPK modeling are listed in clinicaltrial.gov and details of these clinical studies published elsewhere. The data that support the findings of this study are available from supplementary files and repository of PBPK files can be found in Simcyp members area

\section{References}

1. FDA. Enhancing the Diversity of Clinical Trial Populations - Eligibility Criteria, Enrollment Practices, and Trial Designs Guidance for Industry. 2019.

2. Beigel JH, Tomashek KM, Dodd LE, Mehta AK, Zingman BS, Kalil AC, Hohmann E, Chu HY, Luetkemeyer A, Kline S, Lopez de Castilla D, Finberg RW, Dierberg K, Tapson V, Hsieh L, Patterson TF, Paredes R, Sweeney DA, Short WR, Touloumi G, Lye DC, Ohmagari N, Oh MD, Ruiz-Palacios GM, Benfield T, Fatkenheuer G, Kortepeter MG, Atmar RL, Creech CB, Lundgren J, Babiker AG, Pett S, Neaton JD, 
Burgess TH, Bonnett T, Green M, Makowski M, Osinusi A, Nayak S, Lane HC, Members A-SG. Remdesivir for the Treatment of Covid-19 - Preliminary Report. N Engl J Med 2020.

3. Roschewski M, Lionakis MS, Sharman JP, Roswarski J, Goy A, Monticelli MA, Roshon M, Wrzesinski SH, Desai JV, Zarakas MA, Collen J, Rose K, Hamdy A, Izumi R, Wright GW, Chung KK, Baselga J, Staudt LM, Wilson WH. Inhibition of Bruton tyrosine kinase in patients with severe COVID-19. Sci Immunol 2020; 5 .

4. Treon SP, Castillo JJ, Skarbnik AP, Soumerai JD, Ghobrial IM, Guerrera ML, Meid K, Yang G. The BTK inhibitor ibrutinib may protect against pulmonary injury in COVID-19-infected patients. Blood 2020; 135: 1912-15.

5. Administration FFD. FDA Guidance on Conduct of Clinical Trials of Medical Products during COVID-19 Public Health Emergency. 2020.

6. Jarvis CI, Van Zandvoort K, Gimma A, Prem K, group CC-w, Klepac P, Rubin GJ, Edmunds WJ. Quantifying the impact of physical distance measures on the transmission of COVID-19 in the UK. BMC Med 2020; 18: 124.

7. Gautret P, Lagier JC, Parola P, Hoang VT, Meddeb L, Mailhe M, Doudier B, Courjon J, Giordanengo V, Vieira VE, Dupont HT, Honore S, Colson P, Chabriere E, La Scola B, Rolain JM, Brouqui P, Raoult D. Hydroxychloroquine and azithromycin as a treatment of COVID-19: results of an open-label non-randomized clinical trial. Int J Antimicrob Agents 2020: 105949.

8. Aziz M, Fatima R, Assaly R. Elevated Interleukin-6 and Severe COVID-19: A Meta-Analysis. J Med Virol 2020.

9. Shimabukuro-Vornhagen A, Gödel P, Subklewe M, Stemmler HJ, Schlößer HA, Schlaak M, Kochanek M, Böll B, von Bergwelt-Baildon MSJJfioc. Cytokine release syndrome. 2018; 6: 56.

10. Singhal T. A Review of Coronavirus Disease-2019 (COVID-19). Indian J Pediatr 2020; 87: 281-86.

11. Ye Q, Wang B, Mao JJTJoi. The pathogenesis and treatment of theCytokine Storm'in COVID-19. 2020.

12. Schwenger E, Reddy VP, Moorthy G, Sharma P, Tomkinson H, Masson E, Vishwanathan K. Harnessing Meta-analysis to Refine an Oncology Patient Population for Physiology-Based Pharmacokinetic Modeling of Drugs. Clin Pharmacol Ther 2018; 103: 271-80.

13. Pilla Reddy V, Bui K, Scarfe G, Zhou D, Learoyd M. Physiologically Based Pharmacokinetic Modeling for Olaparib Dosing Recommendations: Bridging Formulations, Drug Interactions, and Patient Populations. Clin Pharmacol Ther 2019; 105: 229-41.

14. Gaohua L, Wedagedera J, Small BG, Almond L, Romero K, Hermann D, Hanna D, Jamei M, Gardner I. Development of a Multicompartment Permeability-Limited Lung PBPK Model and Its Application in Predicting Pulmonary Pharmacokinetics of Antituberculosis Drugs. CPT Pharmacometrics Syst Pharmacol 2015; 4: 605-13.

15. (ASHP) ASoH-SP. Assessment of Evidence for COVID-19-Related Treatments. <https://www.ashp.org//media/assets/pharmacy-practice/resource-centers/Coronavirus/docs/ASHP-COVID-19-Evidence-Table>.

16. IQ. Industry Perspectives on Approaches to Evaluate the Effect of Renal Impairment on Drug Exposure. 2018.

17. Rowland Yeo K, Zhang M, Pan X, Ban Ke A, Jones HM, Wesche D, Almond LM. Impact of disease on plasma and lung exposure of chloroquine, hydroxy-chloroquine and azithromycin: application of PBPK modelling. Clin Pharmacol Ther 2020.

18. Williams SJ, Baird-Lambert JA, Farrell GC. Inhibition of theophylline metabolism by interferon. Lancet 1987; 2: 939-41. 
19. Schmitt C, Kuhn B, Zhang X, Kivitz AJ, Grange S. Disease-drug-drug interaction involving tocilizumab and simvastatin in patients with rheumatoid arthritis. Clin Pharmacol Ther 2011; 89: 735-40.

20. Lee EB, Daskalakis N, Xu C, Paccaly A, Miller B, Fleischmann R, Bodrug I, Kivitz A. Disease-Drug Interaction of Sarilumab and Simvastatin in Patients with Rheumatoid Arthritis. Clin Pharmacokinet 2017; 56: $607-15$.

21. Keller R, Klein M, Thomas M, Drager A, Metzger U, Templin MF, Joos TO, Thasler WE, Zell A, Zanger UM. Coordinating Role of RXRalpha in Downregulating Hepatic Detoxification during Inflammation Revealed by Fuzzy-Logic Modeling. PLoS Comput Biol 2016; 12: e1004431.

22. Roytblat L, Rachinsky M, Fisher A, Greemberg L, Shapira Y, Douvdevani A, Gelman S. Raised interleukin-6 levels in obese patients. Obes Res 2000; 8: 673-5.

23. Hidaka T, Suzuki K, Kawakami M, Okada M, Kataharada K, Shinohara T, Takamizawa-Matsumoto M, Ohsuzu F. Dynamic changes in cytokine levels in serum and synovial fluid following filtration leukocytapheresis therapy in patients with rheumatoid arthritis. J Clin Apher 2001; 16: 74-81.

24. Arican O, Aral M, Sasmaz S, Ciragil P. Serum levels of TNF-alpha, IFN-gamma, IL-6, IL-8, IL-12, IL-17, and IL-18 in patients with active psoriasis and correlation with disease severity. Mediators Inflamm 2005; 2005: 273-9.

25. Ataseven H, Bahcecioglu IH, Kuzu N, Yalniz M, Celebi S, Erensoy A, Ustundag B. The levels of ghrelin, leptin, TNF-alpha, and IL-6 in liver cirrhosis and hepatocellular carcinoma due to HBV and HDV infection. Mediators Inflamm 2006; 2006: 78380.

26. Fan J, Zhang X, Liu J, Yang Y, Zheng N, Liu Q, Bergman K, Reynolds K, Huang SM, Zhu H, Wang Y. Connecting hydroxychloroquine in vitro antiviral activity to in vivo concentration for prediction of antiviral effect: a critical step in treating COVID-19 patients. Clin Infect Dis 2020.

27. Jeon S, Ko M, Lee J, Choi I, Byun SY, Park S, Shum D, Kim S. Identification of antiviral drug candidates against SARS-CoV-2 from FDA-approved drugs. Antimicrob Agents Chemother 2020.

28. Ufuk A, Assmus F, Francis L, Plumb J, Damian V, Gertz M, Houston JB, Galetin A. In Vitro and in Silico Tools To Assess Extent of Cellular Uptake and Lysosomal Sequestration of Respiratory Drugs in Human Alveolar Macrophages. Mol Pharm 2017; 14: 1033-46.

29. Pilla Reddy V, Walker M, Sharma P, Ballard P, Vishwanathan K. Development, Verification, and Prediction of Osimertinib Drug-Drug Interactions Using PBPK Modeling Approach to Inform Drug Label. CPT Pharmacometrics Syst Pharmacol 2018; 7: 321-30.

30. Shebley M, Sandhu P, Emami Riedmaier A, Jamei M, Narayanan R, Patel A, Peters SA, Reddy VP, Zheng M, de Zwart L, Beneton M, Bouzom F, Chen J, Chen Y, Cleary Y, Collins C, Dickinson GL, Djebli N, Einolf HJ, Gardner I, Huth F, Kazmi F, Khalil F, Lin J, Odinecs A, Patel C, Rong H, Schuck E, Sharma P, Wu SP, Xu Y, Yamazaki S, Yoshida K, Rowland M. Physiologically Based Pharmacokinetic Model Qualification and Reporting Procedures for Regulatory Submissions: A Consortium Perspective. Clin Pharmacol Ther 2018; 104: 88-110.

31. Taskar KS, Pilla Reddy V, Burt H, Posada MM, Varma M, Zheng M, Ullah M, Emami Riedmaier A, Umehara KI, Snoeys J, Nakakariya M, Chu X, Beneton M, Chen Y, Huth F, Narayanan R, Mukherjee D, Dixit V, Sugiyama Y, Neuhoff S. Physiologically-Based Pharmacokinetic Models for Evaluating Membrane Transporter Mediated Drug-Drug Interactions: Current Capabilities, Case Studies, Future Opportunities, and Recommendations. Clin Pharmacol Ther 2020; 107: 1082-115.

32. Verscheijden LFM, van der Zanden TM, van Bussel LPM, de Hoop-Sommen M, Russel FGM, Johnson TN, de Wildt SN. Chloroquine dosing recommendations for pediatric COVID-19 supported by modeling and simulation. Clin Pharmacol Ther 2020. 
33. Cao B, Wang Y, Wen D, Liu W, Wang J, Fan G, Ruan L, Song B, Cai Y, Wei M, Li X, Xia J, Chen N, Xiang J, Yu T, Bai T, Xie X, Zhang L, Li C, Yuan Y, Chen H, Li H, Huang H, Tu S, Gong F, Liu Y, Wei Y, Dong C, Zhou F, Gu X, Xu J, Liu Z, Zhang Y, Li H, Shang L, Wang K, Li K, Zhou X, Dong X, Qu Z, Lu S, Hu X, Ruan S, Luo S, Wu J, Peng L, Cheng F, Pan L, Zou J, Jia C, Wang J, Liu X, Wang S, Wu X, Ge Q, He J, Zhan H, Qiu F, Guo L, Huang C, Jaki T, Hayden FG, Horby PW, Zhang D, Wang C. A Trial of Lopinavir-Ritonavir in Adults Hospitalized with Severe Covid-19. N Engl J Med 2020; 382: 1787-99.

34. Pilla Reddy V, Anjum R, Grondine M, Smith A, Bhavsar D, Barry E, Guichard SM, Shao W, Kettle JG, Brown C, Banks E, Jones RDO. The pharmacokinetic-pharmacodynamic (PKPD) relationships of AZD3229, a novel and selective inhibitor of cKIT, in a range of mouse xenograft models of GIST. Clin Cancer Res 2020 .

35. Retallack H, Di Lullo E, Arias C, Knopp KA, Laurie MT, Sandoval-Espinosa C, Mancia Leon WR, Krencik R, Ullian EM, Spatazza J, Pollen AA, Mandel-Brehm C, Nowakowski TJ, Kriegstein AR, DeRisi JL. Zika virus cell tropism in the developing human brain and inhibition by azithromycin. Proc Natl Acad Sci U S A 2016; 113: 14408-13.

36. Gielen V, Johnston SL, Edwards MR. Azithromycin induces anti-viral responses in bronchial epithelial cells. Eur Respir J 2010; 36: 646-54.

37. Bacharier LB, Guilbert TW, Mauger DT, Boehmer S, Beigelman A, Fitzpatrick AM, Jackson DJ, Baxi SN, Benson M, Burnham CD, Cabana M, Castro M, Chmiel JF, Covar R, Daines M, Gaffin JM, Gentile DA, Holguin F, Israel E, Kelly HW, Lazarus SC, Lemanske RF, Jr., Ly N, Meade K, Morgan W, Moy J, Olin T, Peters SP, Phipatanakul W, Pongracic JA, Raissy HH, Ross K, Sheehan WJ, Sorkness C, Szefler SJ, Teague WG, Thyne S, Martinez FD. Early Administration of Azithromycin and Prevention of Severe Lower Respiratory Tract Illnesses in Preschool Children With a History of Such Illnesses: A Randomized Clinical Trial. JAMA 2015; 314: 2034-44.

38. USA FDA. HIGHLIGHTS OF PRESCRIBING INFORMATION: XOFLUZATM (baloxavir marboxil) tablets, for oral use. In: FDA US Food \& Drug Administration, October 2019.

39. Yao X, Ye F, Zhang M, Cui C, Huang B, Niu P, Liu X, Zhao L, Dong E, Song C, Zhan S, Lu R, Li H, Tan W, Liu D. In Vitro Antiviral Activity and Projection of Optimized Dosing Design of Hydroxychloroquine for the Treatment of Severe Acute Respiratory Syndrome Coronavirus 2 (SARS-CoV-2). Clin Infect Dis 2020.

40. Liu J, Cao R, Xu M, Wang X, Zhang H, Hu H, Li Y, Hu Z, Zhong W, Wang M. Hydroxychloroquine, a less toxic derivative of chloroquine, is effective in inhibiting SARS-CoV-2 infection in vitro. Cell Discov 2020; 6: 16 .

41. Moon S, Lee S, Kim H, Freitas-Junior LH, Kang M, Ayong L, Hansen MA. An image analysis algorithm for malaria parasite stage classification and viability quantification. PLoS One 2013; 8: e61812.

42. Han S, Hagan DL, Taylor JR, Xin L, Meng W, Biller SA, Wetterau JR, Washburn WN, Whaley JM. Dapagliflozin, a selective SGLT2 inhibitor, improves glucose homeostasis in normal and diabetic rats. Diabetes 2008; 57: 1723-9.

43. Lv Z, Chu Y, Wang Y. HIV protease inhibitors: a review of molecular selectivity and toxicity. HIV AIDS (Auckl) 2015; 7: 95-104.

44. Fintelman-Rodrigues N, Sacramento CQ, Lima CR, da Silva FS, Ferreira AC, Mattos M, de Freitas CS, Soares VC, Gomes Dias SdS, Temerozo JR, Miranda M, Matos AR, Bozza FA, Carels N, Alves CR, Siqueira MM, Bozza PT, Souza TML. 2020.

45. Richardson P, Griffin I, Tucker C, Smith D, Oechsle O, Phelan A, Stebbing JJL. Baricitinib as potential treatment for 2019-nCoV acute respiratory disease. 2020; 395: e30.

46. Roskoski R, Jr. Janus kinase (JAK) inhibitors in the treatment of inflammatory and neoplastic diseases. Pharmacol Res 2016; 111: 784-803. 
47. Warren TK, Jordan R, Lo MK, Ray AS, Mackman RL, Soloveva V, Siegel D, Perron M, Bannister R, Hui HC, Larson N, Strickley R, Wells J, Stuthman KS, Van Tongeren SA, Garza NL, Donnelly G, Shurtleff AC, Retterer CJ, Gharaibeh D, Zamani R, Kenny T, Eaton BP, Grimes E, Welch LS, Gomba L, Wilhelmsen CL, Nichols DK, Nuss JE, Nagle ER, Kugelman JR, Palacios G, Doerffler E, Neville S, Carra E, Clarke MO, Zhang L, Lew W, Ross B, Wang Q, Chun K, Wolfe L, Babusis D, Park Y, Stray KM, Trancheva I, Feng JY, Barauskas O, Xu Y, Wong P, Braun MR, Flint M, McMullan LK, Chen SS, Fearns R, Swaminathan S, Mayers DL, Spiropoulou CF, Lee WA, Nichol ST, Cihlar T, Bavari S. Therapeutic efficacy of the small molecule GS-5734 against Ebola virus in rhesus monkeys. Nature 2016; 531: 381-5.

48. McMullan LK, Flint M, Chakrabarti A, Guerrero L, Lo MK, Porter D, Nichol ST, Spiropoulou CF, Albarino C. Characterisation of infectious Ebola virus from the ongoing outbreak to guide response activities in the Democratic Republic of the Congo: a phylogenetic and in vitro analysis. Lancet Infect Dis 2019; 19: 1023-32.

49. Siegel D, Hui HC, Doerffler E, Clarke MO, Chun K, Zhang L, Neville S, Carra E, Lew W, Ross B, Wang Q, Wolfe L, Jordan R, Soloveva V, Knox J, Perry J, Perron M, Stray KM, Barauskas O, Feng JY, Xu Y, Lee G, Rheingold AL, Ray AS, Bannister R, Strickley R, Swaminathan S, Lee WA, Bavari S, Cihlar T, Lo MK, Warren TK, Mackman RL. Discovery and Synthesis of a Phosphoramidate Prodrug of a Pyrrolo[2,1f] [triazin-4-amino] Adenine C-Nucleoside (GS-5734) for the Treatment of Ebola and Emerging Viruses. J Med Chem 2017; 60: 1648-61.

50. Yu R, Song D, DuBois DC, Almon RR, Jusko WJ. Modeling Combined Anti-Inflammatory Effects of Dexamethasone and Tofacitinib in Arthritic Rats. AAPS J 2019; 21: 93.

51. Loew D, Schuster O, Graul EH. Dose-dependent pharmacokinetics of dexamethasone. Eur J Clin Pharmacol 1986; 30: 225-30.

52. USA FDA. < Tocilizumab FDA label.pdf >.

53. Abdallah H, Hsu JC, Lu P, Fettner S, Zhang X, Douglass W, Bao M, Rowell L, Burmester GR, Kivitz A. Pharmacokinetic and Pharmacodynamic Analysis of Subcutaneous Tocilizumab in Patients With Rheumatoid Arthritis From 2 Randomized, Controlled Trials: SUMMACTA and BREVACTA. J Clin Pharmacol 2017; 57: 459-68.

54. Mihara M, Ohsugi Y, Kishimoto T. Tocilizumab, a humanized anti-interleukin-6 receptor antibody, for treatment of rheumatoid arthritis. Open Access Rheumatol 2011; 3: 19-29.

55. Khan F, Fabbri L, Stewart I, Robinson K, Smyth AR, Jenkins G. 2020.

56. Hensley LE, Fritz LE, Jahrling PB, Karp CL, Huggins JW, Geisbert TW. Interferon-beta 1a and SARS coronavirus replication. Emerg Infect Dis 2004; 10: 317-9.

57. USA FDA, Anakinra. https://www.accessdata.fda.gov/drugsatfda_docs/nda/2001/103950-0_Kineret_Biopharmr.PDF. 2001.

58. USA FDA, Siltuximab. https://www.accessdata.fda.gov/drugsatfda_docs/nda/2014/125496Orig1s000MedR.pdf.

59. USA FDA. Highlights of prescribing information for Calquence (acalabrutinib) capsules, for oral use. In, 2017.

60. USA FDA. Highlights of prescribing information for Zithromax (azithromycin) for IV infusion only. In, 2017.

61. USA FDA. Highlights of prescribing information: Olumiant (baricitinib) tablets, for oral use. In, 2018.

62. USA FDA. Highlights of Prescribing Information: Xofluza (baloxavir marboxil). In, 2018.

63. USA FDA. ARALEN Chloroquine Phosphate, USP Label. In, 2018. 
64. USA FDA. PLAQUENIL Hydroxychloroquine sulfate tablets, USP. In, 2019.

65. USA FDA Darunavir. https://www.accessdata.fda.gov/drugsatfda_docs/label/2017/021976s045_202895s020lbl.pdf. 2017.

66. USA FDA. Highlights of prescribing information for FARXIGA (dapagliflozin). In, January 2020.

67. de Zwart L, Snoeys J, De Jong J, Sukbuntherng J, Mannaert E, Monshouwer M. Ibrutinib Dosing Strategies Based on Interaction Potential of CYP3A4 Perpetrators Using Physiologically Based Pharmacokinetic Modeling. Clin Pharmacol Ther 2016; 100: 548-57.

68. USA FDA, Ibrutinib. https://www.accessdata.fda.gov/drugsatfda_docs/label/2018/210563s000lbl.pdf. 2018.

69. USA FDA. Fact sheet for health care providers: Emergency use authorization (EUA) of Remdesivir (GS-5734). In, 2020, May 1.

70. USA FDA. Highlights of prescribing information for JAKAFI (ruxolitinib) tablets, for oral use. In, 2011.

71. USA FDA. Highlights of prescribing information for NORVIR (ritonavir) tablet, for oral use/ oral solution/ oral powder. In, 2017.

72. USA FDA. NDA/BLA Multi-disciplinary Review and Evaluation \{NDA 210259\} \{CALQUENCE, acalabrutinib\}. In, edResearch CfDEa, FDA US Food \& Drug Administration, 2016, February 1.

73. de Jong J, Skee D, Hellemans P, Jiao J, de Vries R, Swerts D, Lawitz E, Marbury T, Smith W, Sukbuntherng J, Mannaert E. Single-dose pharmacokinetics of ibrutinib in subjects with varying degrees of hepatic impairment<sup/>. Leuk Lymphoma 2017; 58: 185-94.

74. Mazzei T, Surrenti C, Novelli A, Crispo A, Fallani S, Carla V, Surrenti E, Periti P. Pharmacokinetics of azithromycin in patients with impaired hepatic function. J Antimicrob Chemother 1993; 31 Suppl E: 57-63.

75. Gustafsson LL, Walker O, Alvan G, Beermann B, Estevez F, Gleisner L, Lindstrom B, Sjoqvist F. Disposition of chloroquine in man after single intravenous and oral doses. Br J Clin Pharmacol 1983; 15: 471-9.

76. Tett SE, Cutler DJ, Day RO, Brown KF. Bioavailability of hydroxychloroquine tablets in healthy volunteers. Br J Clin Pharmacol 1989; 27: 771-9.

77. Baricitinib. NDA/BLA Multi-disciplinary Review and Evaluation \{NDA 20792\} \{Baricitinib\}.

78. Peng JZ, Pulido F, Causemaker SJ, Li J, Lorenzo A, Cepeda C, Garcia Cabanillas JA, DaSilva B, Brun SC, Arribas J. Pharmacokinetics of lopinavir/ritonavir in HIV/hepatitis C virus-coinfected subjects with hepatic impairment. J Clin Pharmacol 2006; 46: 265-74.

79. USA FDA Atazanavir. https://www.accessdata.fda.gov/drugsatfda_docs/label/2005/021567s005lbl.pdf.

80. Sekar V, Spinosa-Guzman S, De Paepe E, Stevens T, Tomaka F, De Pauw M, Hoetelmans RM. Pharmacokinetics of multiple-dose darunavir in combination with low-dose ritonavir in individuals with mild-tomoderate hepatic impairment. Clin Pharmacokinet 2010; 49: 343-50.

81. Chen X, Shi JG, Emm T, Scherle PA, McGee RF, Lo Y, Landman RR, Punwani NG, Williams WV, Yeleswaram S. Pharmacokinetics and pharmacodynamics of orally administered ruxolitinib (INCB018424 phosphate) in renal and hepatic impairment patients. Clin Pharmacol Drug Dev 2014; 3: 34-42.

82. USA FDA. Highlights of prescribing information for Dexamethsone (Hemady).

83. Hoffler D, Koeppe P, Paeske B. Pharmacokinetics of azithromycin in normal and impaired renal function. Infection 1995; 23: 356-61. 
84. Salako LA, Walker O, Iyun AO. Pharmacokinetics of chloroquine in renal insufficiency. Afr J Med Med Sci 1984; 13: 177-82.

85. Kasichayanula S, Liu X, Pe Benito M, Yao M, Pfister M, LaCreta FP, Humphreys WG, Boulton DW. The influence of kidney function on dapagliflozin exposure, metabolism and pharmacodynamics in healthy subjects and in patients with type 2 diabetes mellitus. Br J Clin Pharmacol 2013; 76: 432-44.

86. Lopinavir. https://liverpool-hiv-hep.s3.amazonaws.com/prescribing_resources/pdfs/000/000/073/original/HIV_FactSheet_LPV_2016_Mar.pdf?1520612690 2016

\section{Figure legends}

Figure 1: Symptoms and grades of cytokine release syndrome, also known as cytokine storm and its impact on drug exposure

Figure 2. Effect of age (A) and Race (B) on plasma concentration -time profiles of COVID-19 drugs

The black continuous line represents the median prediction using the PBPK model for 18-40yrs of age; green line for 40 to 65 years and red line for 65 to 98 years of age. The shaded area represents the $95 \%$ prediction intervals of Caucasian healthy volunteers. In Figure 2B, the black continuous line represents the median prediction using the PBPK model for Caucasians subjects. green line is for Japanese subjects and red line for Chinese patients. All these effect of Race simulations were run using 40 to 65 years of age with $50 \%$ of females.

Doses used: Acalabrutinib 100mg single dose, azithromycin 500mg single dose, chloroquine $600 \mathrm{mg}$ single dose, dapagliflozin 10mg single dose, darunavir 800mg single dose, hydroxychloroquine (HCQ) 200mg single dose, ibrutinib $140 \mathrm{mg}$ single dose; lopinavir 400mg single dose (with 100mg ritonavir concomitant interaction, rifampicin $600 \mathrm{mg}$ single dose, baricitinib $2 \mathrm{mg}$ single dose, ritonavir 600mg single dose, ruxolitinib 20mg single dose.

Figure 3. Effect of hepatic (A) and renal (B) impairments on plasma concentration-time profiles of COVID-19 drugs

The black continuous line represents the median prediction using the PBPK model for healthy population. The shaded area represents the $95 \%$ prediction intervals of healthy population. Blue line represents mild hepatic impairment. Green line represents moderate renal or hepatic impairment; red line represents severe renal or hepatic impairment.

Doses used for hepatic impairment: Acalabrutinib $50 \mathrm{mg}$ single dose, azithromycin 500mg single dose, atazanavir 400mg single dose, chloroquine 300mg single dose, dapagliflozin 10mg single dose, hydroxychloroquine base(HCQ) $155 \mathrm{mg}$ single dose, baricitinib 4mg single dose, ritonavir 600mg single dose, ruxolitinib 25mg single dose, lopinavir $400 \mathrm{mg}$ single dosing interval (with 100mg ritonavir concomitant interaction), darunavir $600 \mathrm{mg}$ single dosing interval (with $100 \mathrm{mg}$ ritonavir concomitant interaction), ibrutinib 140mg single dose, dexamethasone8 mg single dose. Only parent acalabrutinib was measured in organ dysfunction or DDI studies of acalabrutinib.

Doses used for renal impairment: Azithromycin 500mg single dose, atazanavir 400mg single dose, hydroxychloroquine base $155 \mathrm{mg}$, baricitinib 4mg single dose, chloroquine 300mg single dose, dapagliflozin $50 \mathrm{mg}$ single dose, acalabrutinib $50 \mathrm{mg}$ single dose, ruxolitinib $25 \mathrm{mg}$ single dose, ritonavir $600 \mathrm{mg}$ single dose, lopinavir $400 \mathrm{mg}$ single dose (with 100mg ritonavir concomitant interaction), darunavir $800 \mathrm{mg}$ single dose, dexamethasone $8 \mathrm{mg}$ single dose.

Figure 4. PBPK model-based simulations of conc-profiles in plasma and lung tissue using multiplecompartmental lung model in geriatric patients after verification of models using adult data for drugs that are being tested in COVID-Trial . Dashed lines represents a relevant potency value either $\mathrm{I}_{\mathrm{C} 50}$ or $\mathrm{IC}_{90}$. 
Doses used for simulating lung exposure: multiple doses 14 days of dosing to steady state azithromycin 500 mg single dose, atazanavir $400 \mathrm{mg}$, hydroxychloroquine base $155 \mathrm{mg}$, baricitinib $4 \mathrm{mg}$, chloroquine $300 \mathrm{mg}$, dapagliflozin $10 \mathrm{mg}$, acalabrutinib $100 \mathrm{mg}$, ruxolitinib $25 \mathrm{mg}$, ritonavir $600 \mathrm{mg}$, lopinavir $400 \mathrm{mg}$, darunavir $800 \mathrm{mg}$, dexamethasone $8 \mathrm{mg}$.

Figure 5. Sensitivity analysis on the possible effect of cytokines, and consequent CYP3A4 abundance suppression in both liver and the gut on acalabrutinib (A) and ibrutinib (B) exposures
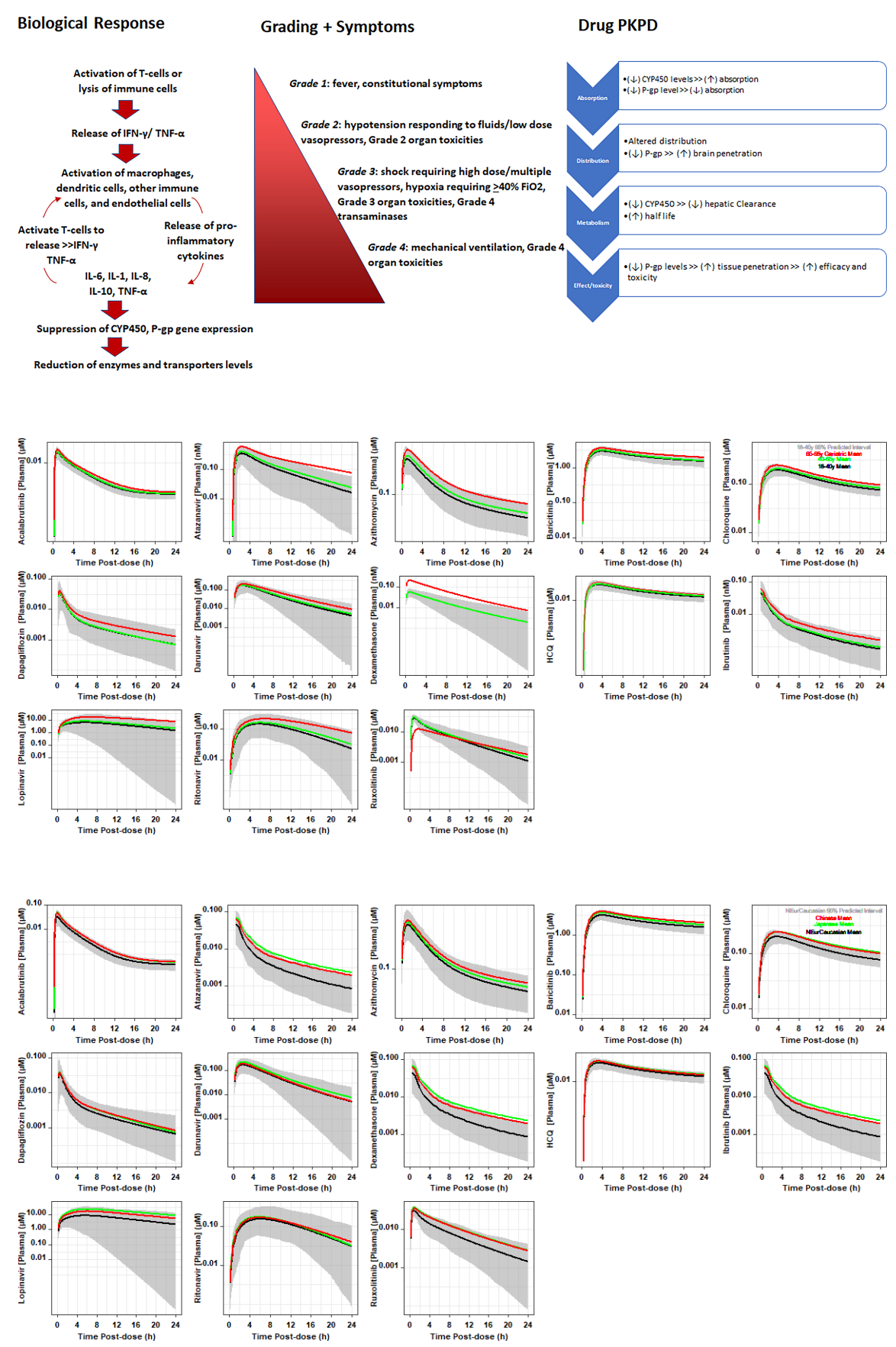

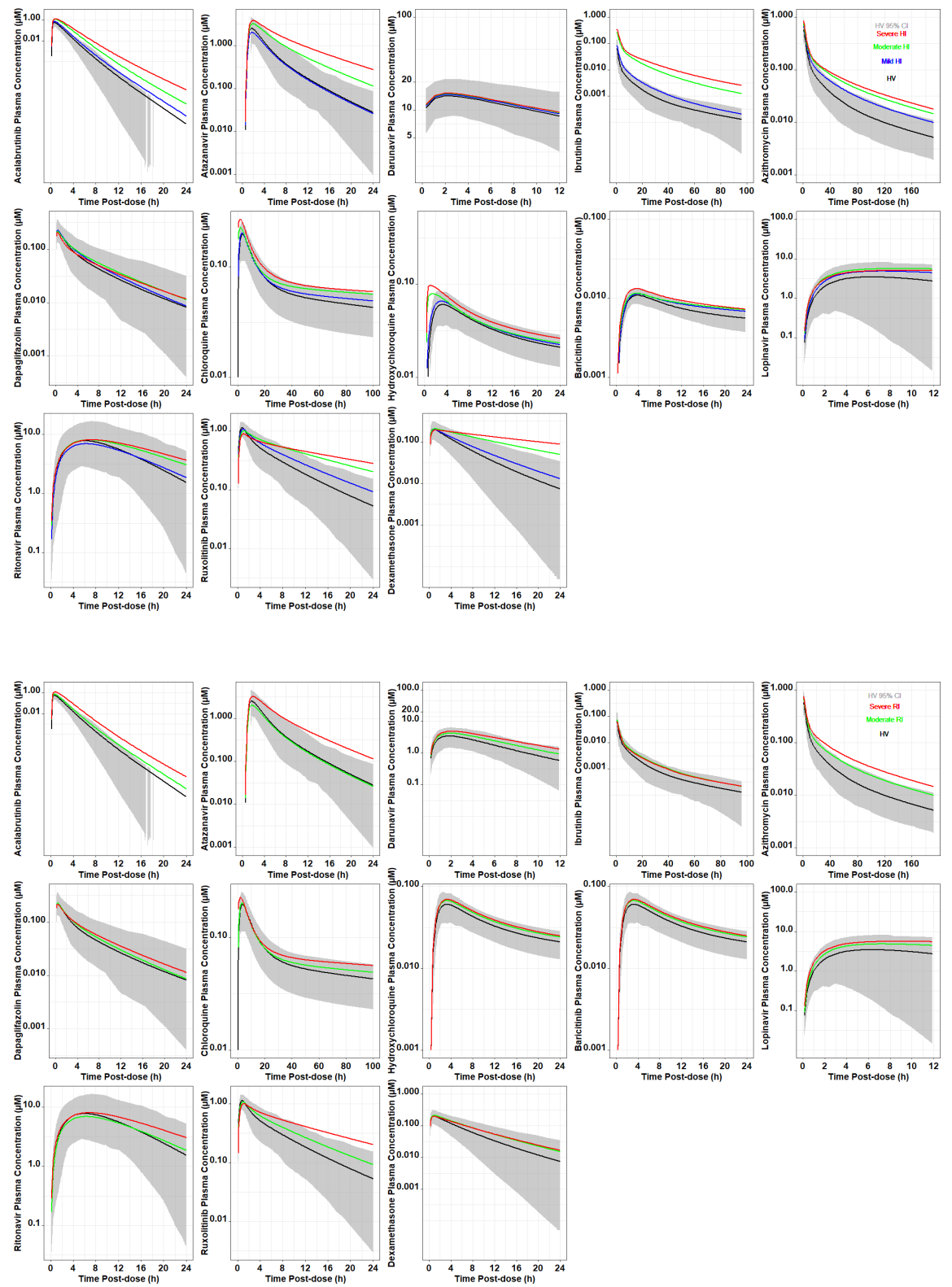

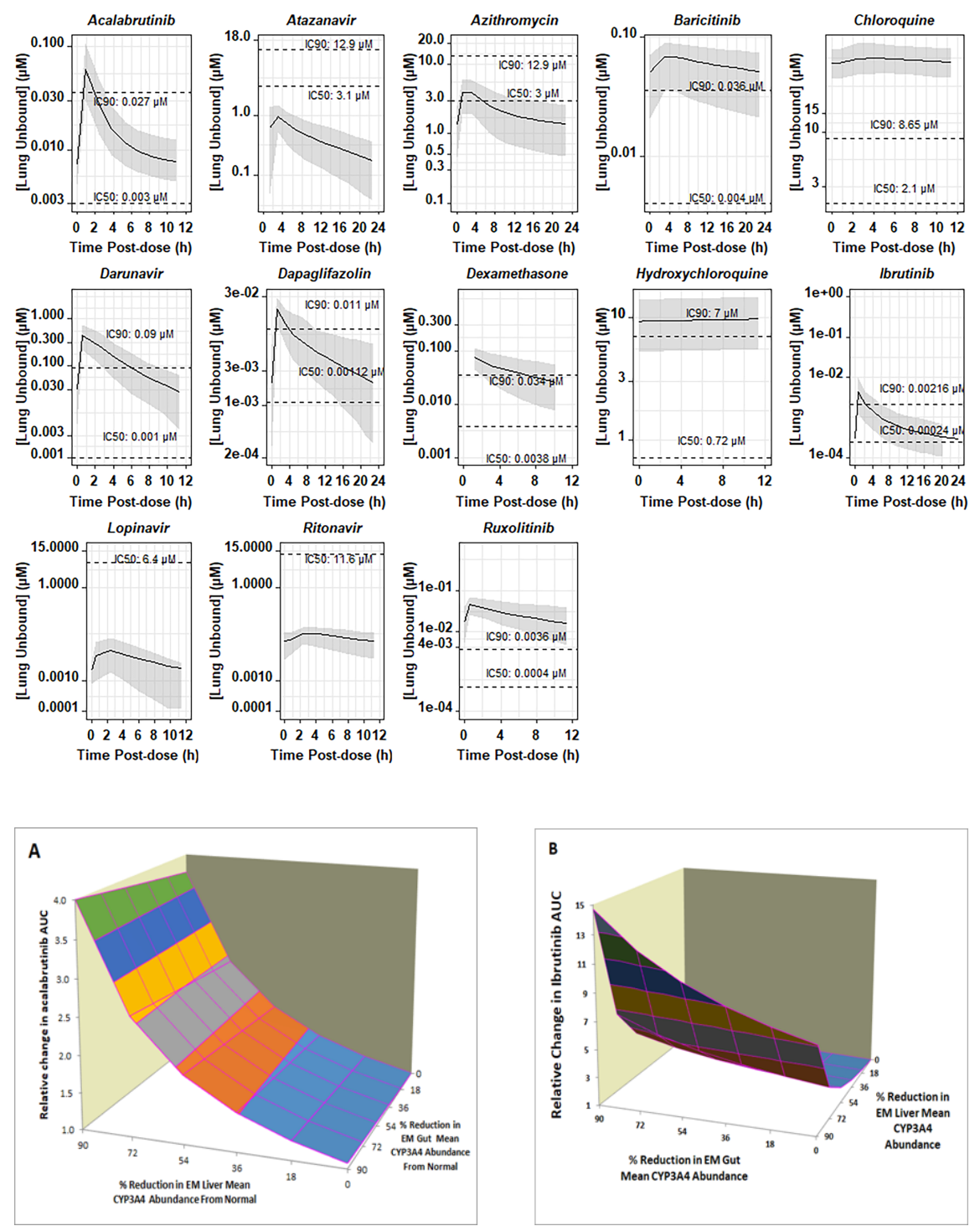

\section{Hosted file}

Table 1.docx available at https://authorea.com/users/337414/articles/463026-pharmacokineticsunder-the-covid-19-storm

\section{Hosted file}

Table 2.docx available at https://authorea.com/users/337414/articles/463026-pharmacokineticsunder-the-covid-19-storm

\section{Hosted file}

Table 3.docx available at https://authorea.com/users/337414/articles/463026-pharmacokineticsunder-the-covid-19-storm

\section{Hosted file}

Table 4.docx available at https://authorea.com/users/337414/articles/463026-pharmacokineticsunder-the-covid-19-storm 